Original Research Paper

\title{
Gadolinium at Low Concentration Suppresses both Osteoclastic and Osteoblastic Activities in the Scales of Goldfish
}

\author{
${ }^{1}$ Nobuo Suzuki, ${ }^{2}$ Kazuki Watanabe, ${ }^{1}$ Aika Sekimoto, ${ }^{1,3}$ Makoto Urata, ${ }^{1,4}$ Mohamed Ibrahim Zanaty, ${ }^{1}$ Toshio \\ Sekiguchi, ${ }^{1}$ Yoichiro Kitani, ${ }^{5}$ Hajime Matsubara, ${ }^{6}$ Ajai Kumar Srivastav and ${ }^{2}$ Atsuhiko Hattori \\ ${ }^{I}$ Noto Marine Laboratory, Institute of Nature and Environmental Technology, \\ Kanazawa University, Ogi, Noto-cho, Ishikawa 927-0553, Japan \\ ${ }^{2}$ Department of Biology, College of Liberal Arts and Sciences, Tokyo Medical and Dental University, \\ Ichikawa, Chiba 272-0827, Japan \\ ${ }^{3}$ Institute of Noto SATOUMI Education Research, Ogi, Noto-cho, Ishikawa 927-0553, Japan \\ ${ }^{4}$ Biotechnology and Life Sciences Department, Faculty of Postgraduate Studies for Advanced Sciences, \\ Beni-Suef University, Beni-Suef, Egypt \\ ${ }^{5}$ Noto Center for Fisheries Science and Technology, Kanazawa University, Ossaka, Noto-cho, \\ Ishikawa 927-0552, Japan \\ ${ }^{6}$ Department of Zoology, D.D.U. Gorakhpur University, Gorakhpur 273-009, India
}

Article history

Received: 11-08-2019

Revised: 02-09-2019

Accepted: 12-09-2019

Corresponding Author:

Nobuo Suzuki

Noto Marine Laboratory,

Institute of Nature and

Environmental Technology,

Kanazawa University, Ogi,

Noto-cho, Ishikawa 927-0553, Japan

Email: nobuos@staff.kanazawa-u.ac.jp
Abstract: For determining the effect of environmental pollutants on fish bone metabolism, we have developed an in vitro bioassay system using teleost scales that has osteoclasts, osteoblasts and bone matrix as markers: alkaline phosphatase (ALP) for osteoblasts and tartrate-resistant acid phosphatase (TRAP) for osteoclasts. Using this bioassay, the influence of gadolinium $(\mathrm{Gd})$ on osteoclasts and osteoblasts of goldfish scales was examined in the present study. Gd sensitively inhibited TRAP activity. Even Gd at $10^{-13} \mathrm{M}$ suppressed TRAP activity at 6 hours of incubation. At 18 hours of incubation, this inhibition occurred only at $10^{-7}$ and $10^{-6} \mathrm{M}$. After 36 hours of incubation, Gd did not influence TRAP activity. In osteoblasts, ALP activity was also suppressed by Gd in the range of $10^{-10}$ to $10^{-6} \mathrm{M}$ for 6 to 18 hours of incubation. At 36 and 64 hours of incubation, ALP activity was significantly suppressed by Gd (36 hours: $10^{-9}$ to $10^{-6} \mathrm{M}$; 64 hours: $10^{-7}$ and $10^{-6} \mathrm{M}$ ). At 96 hours of incubation, however, Gd did not influence ALP activity. This is the first report to indicate the toxicity of Gd on fish bone metabolism using TRAP and ALP enzyme activities. The toxicity of Gd to osteoblasts is comparable to that of tributyltin, an aquatic environmental pollutant used as a biocide in anti-fouling paint. Gd is used in Magnetic Resonance Imaging (MRI) for clinical diagnoses. To avoid the toxicity of $\mathrm{Gd}$ ions, chelated forms, known as Gd-based contrast agents, are used for MRI diagnosis. Without a specific recycling process, these compounds are quickly released by urinary excretion and released into environmental waters. Therefore, it is possible that anthropogenic Gd influences aquatic animals. Considering our present data together with that of anthropogenic Gd pollution, we should conduct a Gd risk assessment to protect the ecosystem in the aquatic environment.

Keywords: Gadolinium, Osteoclasts, Osteoblasts, Fish Scales, Goldfish

\section{Introduction}

Gadolinium $(\mathrm{Gd})$ is a ductile rare-earth metal. $\mathrm{Gd}^{3+}$ is currently used in magnetic resonance imaging (MRI) for clinical diagnoses because $\mathrm{Gd}^{3+}$ has paramagnetic properties (Möller et al., 2002; Birka et al., 2016). To avoid the toxicity of $\mathrm{Gd}^{3+}$, chelated forms, known as $\mathrm{Gd}$ based contrast agents (Gd-CAs), have been used (Möller et al., 2002; Telgmann et al., 2013). In general, Gd-CAs are stable complexes. The agents are 
rapidly eliminated from a patient's body. After excretion, they enter the public sewer and, subsequently, the wastewater treatment plant. Because of their polar or anionic nature, however, the $\mathrm{Gd}$ complexes most likely are neither adsorbed onto surfaces nor by particulate organic matter (Knappe et al., 2005) but are released into environmental water without a specific recycling process (Telgmann et al., 2013; Braun et al., 2018). Therefore, a significant amount of anthropogenic Gd-concentration in surface waters has been reported worldwide (Möller et al., 2002; Telgmann et al., 2013; Birka et al., 2016; Braun et al., 2018). It is possible that the anthropogenic Gd impacts aquatic animals. Gd appears toxic in animals because $\mathrm{Gd}$ functions as a blocker of $\mathrm{Ca}$ channels, causing its ionic radius to be nearly equal to that of $\mathrm{Ca}$ (Sherry et al., 2009). Actually, Gd influences calcified tissue such as sea urchin spicules (Saitoh et al., 2010; Martino et al., 2017; 2018). As Gd affects the skeletal formation of sea urchins, we presumed that Gd affects bone metabolism in fish.

We have developed an original in vitro bioassay system using goldfish scales that has osteoclasts, osteoblasts and bone matrix as markers: alkaline phosphatase (ALP) for osteoblasts and tartrate-resistant acid phosphatase (TRAP) for osteoclasts (Suzuki et al., 2000; Suzuki and Hattori, 2002; 2003; Suzuki et al., 2004). Using this in vitro bioassay, we have reported the toxicity of heavy metals such as cadmium $(\mathrm{Cd})$ and mercury $(\mathrm{Hg})$ on fish bone metabolism. We found that inorganic mercury ( $\mathrm{InHg})\left(10^{-5}\right.$ to $\left.10^{-3} \mathrm{M}\right)$ (Suzuki et al., $2011)$ and methylmercury $(\mathrm{MeHg})\left(10^{-8}\right.$ to $\left.10^{-6} \mathrm{M}\right)$ (Suzuki et al., 2004) significantly inhibited the osteoclastic activity of goldfish scales. $\mathrm{Cd}$ responded very sensitively to osteoclasts. We demonstrate that even at $10^{-13} \mathrm{M}, \mathrm{Cd}$ suppressed osteoclastic activity in goldfish scales at 6 hours of incubation (Suzuki et al., 2004). Thus, in the present study, we examined the effect of $\mathrm{Gd}$ on osteoclastic and osteoblastic activities with this goldfish scale in vitro bioassay.

\section{Materials and Methods}

\section{Animals}

In goldfish (Carassius auratus auratus), we have reported that sensitivity of osteoblastic and osteoclastic activities of scales to calcemic hormones (estrogen and calcitonin) was higher in mature females than in mature males (Suzuki et al., 2000; Yoshikubo et al., 2005). Therefore, female goldfish $(\mathrm{n}=9$, bw $30.33 \pm 1.71 \mathrm{~g})$ were purchased from a commercial source (Higashikawa Fish Farm, Yamatokoriyama, Japan) and used in the scale in vitro bioassay. In addition, all experimental procedures were conducted in accordance with the Guide for the Care and Use of Laboratory Animals of Kanazawa University.
Effects of $G d$ on Osteoclastic Activity in the Cultured Scales of Goldfish at 6 hours of Incubation

The culture medium was prepared. First, we added $1 \%$ penicillin-streptomycin mixture (ICN Biomedicals Inc., Aurora, OH, USA) and HEPES (Research Organics, Inc., Cleveland, OH, USA) (20 mM) to Earle's Minimum Essential Medium (MEM; ICN Biomedicals Inc.). After filtration, the MEM was used in this experiment.

After preparation of the culture medium, goldfish were anesthetized with ethyl 3-aminobenzoate, methanesulfonic acid salt (Sigma-Aldrich, Inc., St. Louis, MO, USA) and the scales on both sides of the body were then removed. The collected scales were incubated in MEM supplemented with gadolinium acetate $\left(\mathrm{Gd}\left(\mathrm{OCH}_{3} \mathrm{CO}\right)_{3} 4 \mathrm{H}_{2} \mathrm{O}\right)$ (Wako Pure Chemicals, Osaka, Japan) $\left(10^{-15}\right.$ to $\left.10^{-6} \mathrm{M}\right)$ and compared with Gdfree medium as a control. The incubation time and temperature were 6 hours and $15^{\circ} \mathrm{C}$, respectively. We have reported the toxicity of $\mathrm{Cd}$ at $15^{\circ} \mathrm{C}$ at 6 hours of incubation (Suzuki et al., 2004). Therefore, these culture conditions were adopted in the present study. After incubation, scales were fixed in $10 \%$ formalin in a 0.05 $\mathrm{M}$ cacodylate buffer ( $\mathrm{pH} 7.4$ ) and then rinsed in distilled water. These scales were kept in a $0.05 \mathrm{M}$ cacodylate buffer at $4{ }^{\circ} \mathrm{C}$ until analysis. We examined the effects of Gd on osteoclasts using TRAP enzyme activity because, in mammals, the effects of hormones and some bioactive substances on osteoclasts have been investigated using TRAP (Vaes, 1988; Suda et al., 1999). Therefore, we used TRAP activity as an indicator of osteoclasts.

TRAP activity was measured as follows. Each scale was transferred to its own well in a 96-well microplate. An aliquot of $200 \mu \mathrm{L}$ of $10 \mathrm{mM}$ para-nitrophenylphosphate and $20 \mathrm{mM}$ tartrate in a $0.1 \mathrm{M}$ sodium acetate buffer ( $\mathrm{pH}$ 5.3) was added to each well. This plate was then incubated at $20^{\circ} \mathrm{C}$ for 30 min while being shaken. After incubation, the reaction was stopped by adding 50 $\mu \mathrm{L}$ of $2 \mathrm{~N} \mathrm{NaOH}$. The $150 \mu \mathrm{L}$ of colored solution was transferred to a new plate and the absorbance was measured at $405 \mathrm{~nm}$. The absorbance was converted to the amount of para-nitrophenol (pNP) produced using a standard curve for pNP (Suzuki and Hattori, 2002).

\section{Effects of Gd on Scale Osteoclastic Activity in goldfish Scales after Long-Term Culture}

To examine the influence of $\mathrm{Gd}$ after long-term incubation, scales extracted from goldfish under anesthesia with ethyl 3-aminobenzoate, methanesulfonic acid salt (Sigma-Aldrich) were incubated in MEM with or without Gd for 18, 36, 64 and $96 \mathrm{~h}$. After incubation, the 
scales were fixed in $10 \%$ formalin in a $0.05 \mathrm{M}$ cacodylate buffer ( $\mathrm{pH} 7.4)$ and then rinsed in distilled water. These scales were kept in a $0.05 \mathrm{M}$ cacodylate buffer at $4^{\circ} \mathrm{C}$ until analysis. Then, TRAP activity was measured as described above.

Effects of $G d$ on Osteoblastic Activity in the Cultured Scales of Goldfish at 6 Hours of Incubation

Goldfish scales were removed under anesthesia with ethyl 3-aminobenzoate, methanesulfonic acid salt (Sigma-Aldrich). Using these collected scales, we examined the effects of Gd on the osteoblasts with ALP as an indicator of osteoblasts (Dimai et al., 1998; Suda $e t$ al., 1999). The removed scales were incubated in MEM with or without Gd for $6 \mathrm{~h}$. After incubation, scales were fixed in $10 \%$ formalin in a $0.05 \mathrm{M}$ cacodylate buffer $(\mathrm{pH}$ 7.4) and then rinsed in distilled water. These scales were kept in a $0.05 \mathrm{M}$ cacodylate buffer at $4^{\circ} \mathrm{C}$ until analysis. The ALP activity was measured as follows.

Each scale was transferred to its own well in a 96well microplate. An aliquot of $200 \mu \mathrm{L}$ of $10 \mathrm{mM}$ paranitrophenyl-phosphate in an alkaline buffer $(100 \mathrm{mM}$ Tris-HCl, $\mathrm{pH} 9.5 ; 1 \mathrm{mM} \mathrm{MgCl} 2$ ) was added to each well. Then, this plate was incubated at $20^{\circ} \mathrm{C}$ for $30 \mathrm{~min}$ while being shaken. After incubation, the reaction was stopped by adding $50 \mu \mathrm{L}$ of $2 \mathrm{~N} \mathrm{NaOH}$. The $150 \mu \mathrm{L}$ of colored solution was transferred to a new plate and the absorbance was measured at $405 \mathrm{~nm}$. The absorbance was converted to the amount of pNP produced using a standard curve for pNP (Suzuki and Hattori, 2002).

\section{Effects of Gd on Scale Osteoblastic Activity in} goldfish Scales after Long-Term Culture

Scales were extracted from goldfish under anesthesia with ethyl 3-aminobenzoate, methanesulfonic acid salt (Sigma-Aldrich). Using these scales, we examined the effect of Gd during long-term incubation. The extracted scales were incubated in MEM with or without Gd for 18, 36, 64, and 96 hours. After incubation, the incubated scales were fixed in $10 \%$ formalin in a $0.05 \mathrm{M}$ cacodylate buffer ( $\mathrm{pH}$ 7.4) and then rinsed with distilled water. These scales were kept in a $0.05 \mathrm{M}$ cacodylate buffer at $4^{\circ} \mathrm{C}$ until analysis. Then, ALP activity was measured as above described.

\section{Statistical Analyses}

All results are expressed as the means $\pm \mathrm{SE}$. The statistical significance between the control and experimental groups was assessed with one-way analysis of variance followed by Bonferroni's test. In all cases, the selected significance level was $p<0.05$.

\section{Results}

Effect of Gd on Osteoclastic Activity as Seen in the Cultured Scales of Goldfish at 6 Hours of Incubation

Gd inhibited TRAP activity at 6 hours of incubation. Gd significantly suppressed TRAP activity. In 3 goldfish used in the present study, the detection limit of Gd was $10^{-13}$ (Fig. 1a), $10^{-11}$ (Fig. 1b) and $10^{-11} \mathrm{M}$ (Fig. 1c).

(a)

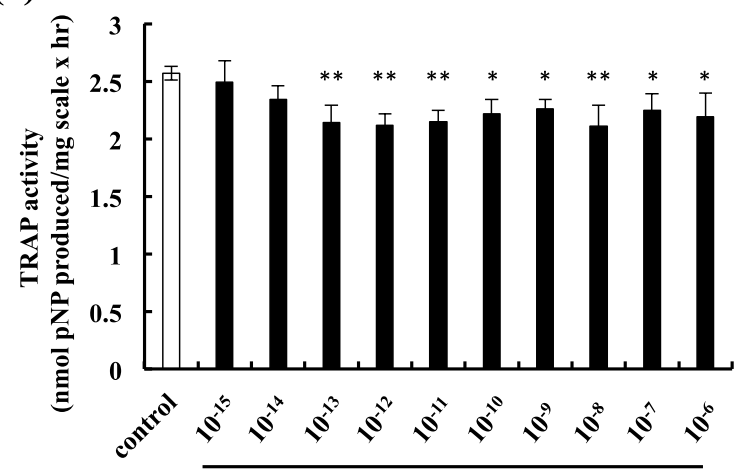

(b)

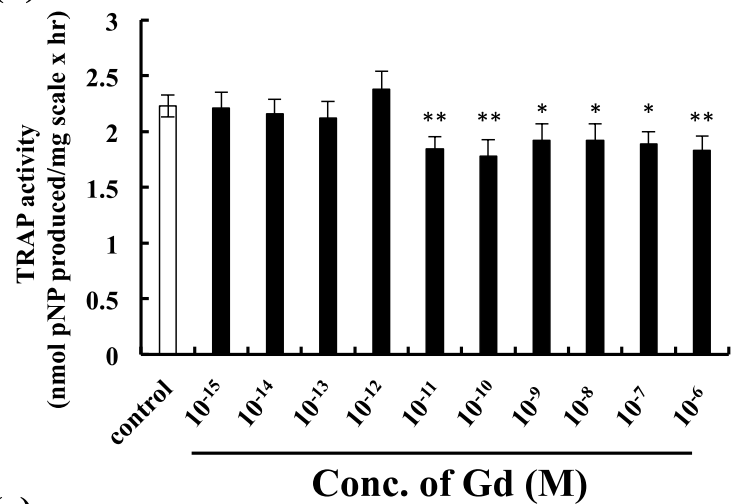

(c)

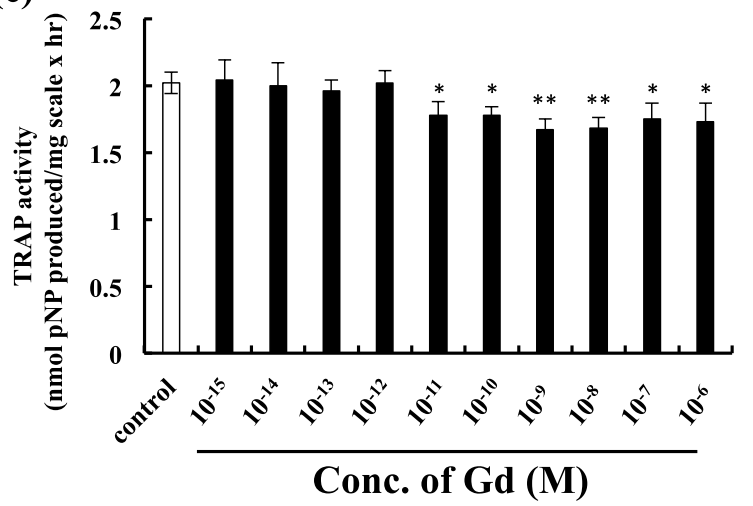

Fig. 1: Effects of Gd on osteoclastic activity in the cultured scales of goldfish at $6 \mathrm{~h}$ of incubation. Results are shown for 3 individual goldfish. * and ** indicate statistically significant differences at $\mathrm{p}<0.05$ and $\mathrm{p}<0.01$, respectively, from the values in the control scales. 
(a) 18 hours incubation

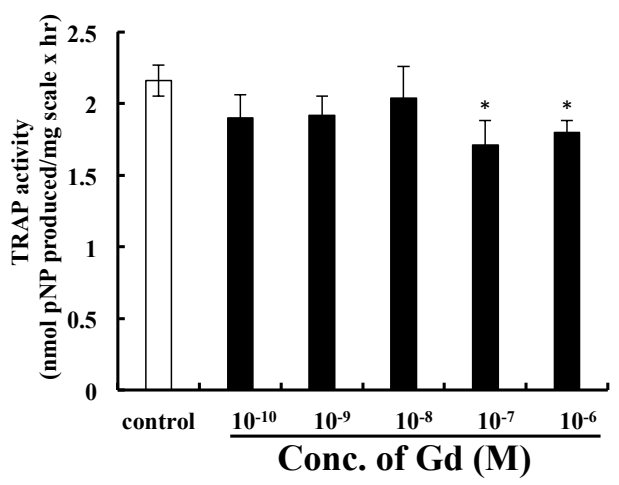

(c) 64 hours incubation

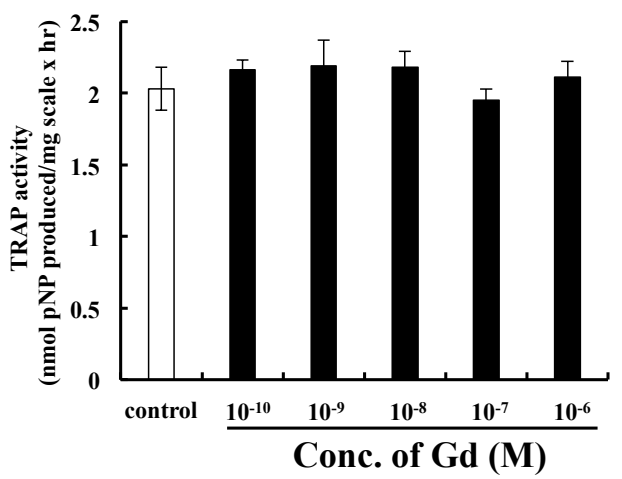

(b) 36 hours incubation

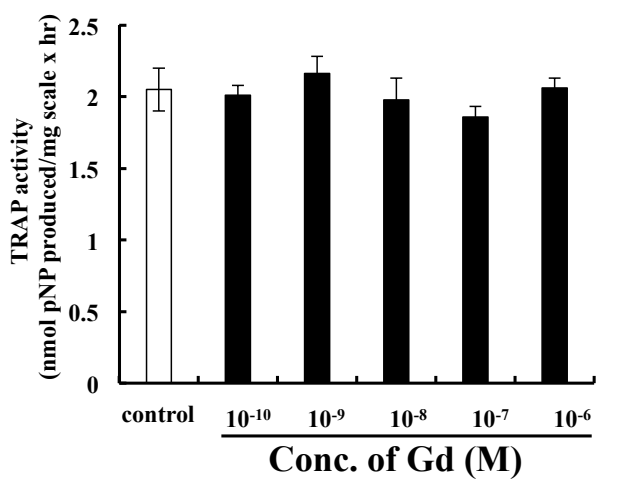

(d) 96 hours incubation

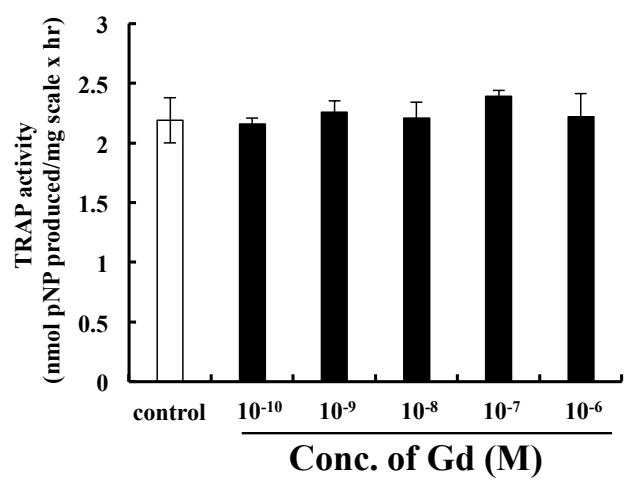

Fig. 2: Effect of Gd on osteoclastic activity after the long-term culture of goldfish scales. * indicates a statistically significant difference at $\mathrm{p}<0.05$ from the values in the control scales.

(a)

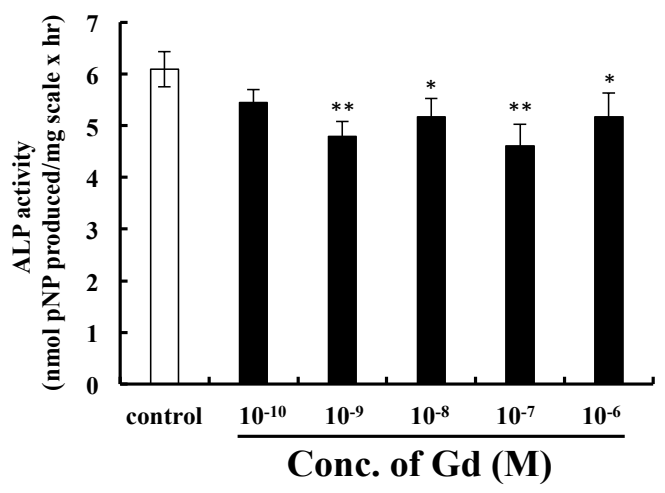

(b)

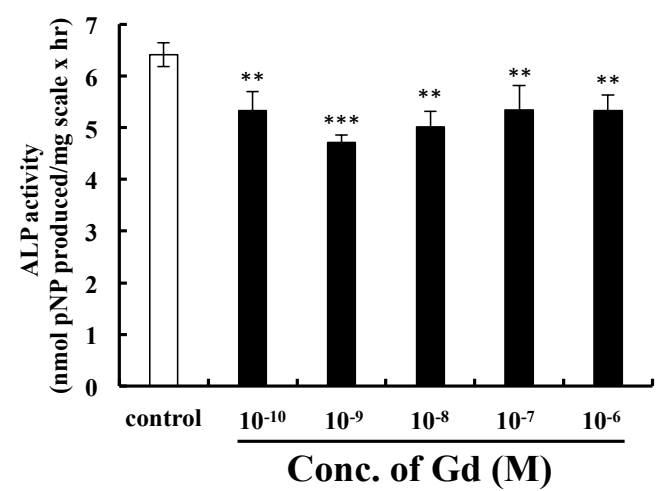

Fig. 3: Effects of Gd on osteoblastic activity in the cultured scales of goldfish at 6 hours of incubation. Results are shown for 2 individual goldfish. *, ** and $* * *$ indicate statistically significant differences at $\mathrm{p}<0.05, \mathrm{p}<0.01$ and $\mathrm{p}<0.001$, respectively, from the values in the control scales.

Effect of Gd on Osteoclastic Activity in the LongTerm Culture of Goldfish Scales

At 18 hours of incubation, TRAP activity was significantly inhibited only at $10^{-7}$ and $10^{-6} \mathrm{M}$ (Fig. 2a). After 36, 64 and 96 hours of incubation, Gd did not influence TRAP activity (Fig. 2b-d).
Effect of Gd on Osteoblastic Activity as Seen in the Cultured Scales of Goldfish at 6 Hours of Incubation

ALP activity was significantly suppressed by Gd (Fig. 3a and b), although the Gd sensitivity was lower than in TRAP. In the 2 goldfish used in the present study, the detection limit of Gd was $10^{-9} \mathrm{M}$ (Fig. 3a) and $10^{-10} \mathrm{M}$ (Fig. 3b). 
(a) 18 hours incubation

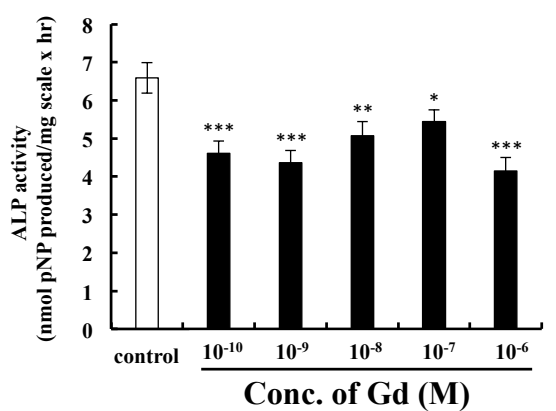

(c) 64 hours incubation

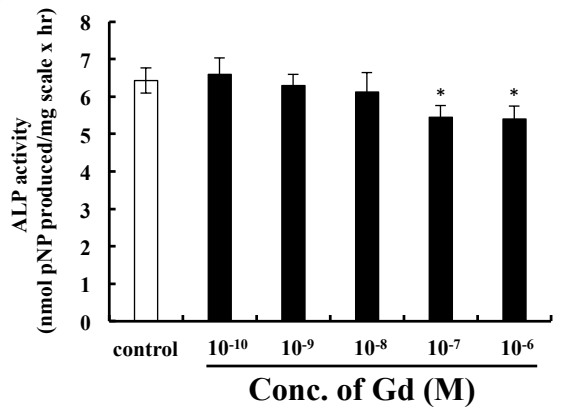

(b) 36 hours incubation

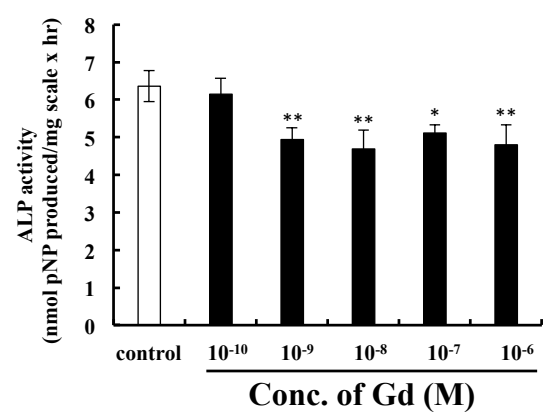

(d) 96 hours incubation

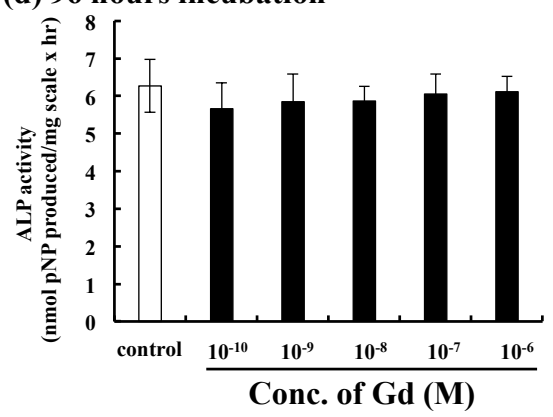

Fig. 4: Effect of Gd on the osteoblastic activity after the long-term culture of goldfish scales. *, ** and *** indicate statistically significant differences at $\mathrm{p}<0.05, \mathrm{p}<0.01$ and $\mathrm{p}<0.001$, respectively, from the values in the control scales.

\section{Effect of Gd on Osteoblastic Activity in the Long- Term Culture of Goldfish Scales}

At 18 hours of incubation, Gd $\left(10^{-10}\right.$ to $\left.10^{-6} \mathrm{M}\right)$ significantly inhibited ALP activity (Fig. 4a). At 36 hours of incubation, ALP activity was significantly suppressed by $\mathrm{Gd}\left(10^{-9}\right.$ to $\left.10^{-6} \mathrm{M}\right)$ (Fig. 4b). This inhibition was maintained at 64 hours of incubation. Gd $\left(10^{-7}\right.$ and $\left.10^{-6} \mathrm{M}\right)$ significantly suppressed ALP activity at 64 hours of incubation (Fig. 4c). However, at $96 \mathrm{~h}$ of incubation, Gd did not influence ALP activity (Fig. 4d).

\section{Discussion}

This is the first report to indicate the toxicity of Gd on fish bone metabolism using TRAP and ALP enzyme activities. In scales as well as mammalian bone, bone $\gamma$ carboxyglutamic acid protein (Nishimoto et al., 1992), osteonectin (Lehane et al., 1999) and osteocalcin (Thamamongood et al., 2012) are present. Thus, the scales of some teleosts are a better potential internal calcium reservoir than vertebral bone during periods of increased calcium demand, such as sexual maturation and starvation (Mugiya and Watabe, 1977; BereiterHahn and Zylberberg, 1993). Therefore, we believe that fish scales are a suitable bone model for the analysis of environmental pollutants. Furthermore, we demonstrated that Gd quite sensitively inhibited TRAP activity. Even Gd of $10^{-13} \mathrm{M}$ suppressed TRAP activity at 6 hours of incubation. In the case of the sea urchin, $\mathrm{Gd}$ in a range of $10^{-7}$ to $10^{-5} \mathrm{M}$ influenced skeletogenesis (Saitoh et al., 2010; Martino et al., 2017 ; 2018). We found that the detection limit of Gd in our bioassay was $10^{-13} \mathrm{M}$. Therefore, our assay system is quite effective as a biosensor for $\mathrm{Gd}$.

At 6 hours of incubation, very low concentrations of Gd $\left(10^{-10}\right.$ and $\left.10^{-9} \mathrm{M}\right)$ influenced osteoblasts and suppressed osteoblastic activity. Therefore, the toxicity of Gd to osteoblasts appears to be higher than that of Cd, $\mathrm{MeHg}$ and $\mathrm{InHg}$ (Suzuki et al., 2004; Suzuki et al., 2011). Heavy metals such as $\mathrm{Cd}, \mathrm{MeHg}$ and $\mathrm{InHg}$ were resistant to each metal as a result of the production of metallothionein (MT), which is a metal-binding protein that protects an organism from heavy metals (Suzuki et al., 2004; 2011). Because the mRNA expression of MT in $\mathrm{Cd}-\mathrm{MeHg}$ - and InHg-treated goldfish scales was increased, osteoblastic activity did not change at 6 hours of incubation (Suzuki et al., 2004; 2011). Thereafter, osteoblastic activity was inhibited by $\mathrm{Cd}$ $\left(10^{-7} \mathrm{M}\right), \mathrm{MeHg}\left(10^{-7} \mathrm{M}\right)$ and $\mathrm{InHg}\left(10^{-6}\right.$ to $\left.10^{-4} \mathrm{M}\right)$ at 36 or 64 hours of incubation (Suzuki et al., 2004; 2011). This phenomenon is also observed in the scales of nibbler fish, Girella punctata (marine fish) (Yachiguchi et al., 2014). ALP activity in the scales of nibbler fish decreased after exposure to $\mathrm{InHg}\left(10^{-5}\right.$ and $\left.10^{-4} \mathrm{M}\right)$ and $\mathrm{MeHg}\left(10^{-6}\right.$ to $\left.10^{-4} \mathrm{M}\right)$ for 18 and 36 hours, although ALP activity did not change after 6 hours of incubation (Yachiguchi et al., 2014). Furthermore, we previously analyzed the toxicity 
Table 1: Toxicity of environmental pollutants on osteoclasts (OC) and osteoblasts (OB) in the cultured scales of teleosts

\begin{tabular}{lllll}
\hline Pollutants & Detection Limit & Target & Name of Teteost & References \\
\hline $\mathrm{Cd}$ & $10^{-13} \mathrm{M}$ & OC & goldfish & Suzuki et al. $(2004)$ \\
$\mathrm{Cd}$ & $10^{-7} \mathrm{M}$ & OB & goldfish & Suzuki et al. $(2004)$ \\
$\mathrm{MeHg}$ & $10^{-8} \mathrm{M}$ & OC & goldfish & Suzuki et al. $(2004)$ \\
$\mathrm{MeHg}$ & $10^{-7} \mathrm{M}$ & OB & goldfish & Suzuki et al. $(2004)$ \\
$\mathrm{InHg}$ & $10^{-5} \mathrm{M}$ & OC & goldfish & Suzuki et al. $(2011)$ \\
$\mathrm{InHg}$ & $10^{-6} \mathrm{M}$ & OB & goldfish & Suzuki et al. $(2011)$ \\
$\mathrm{MeHg}$ & $10^{-6} \mathrm{M}$ & OC & nibbler fish & Yachiguchi et al. $(2014)$ \\
$\mathrm{MeHg}$ & $10^{-6} \mathrm{M}$ & OB & nibbler fish & Yachiguchi et al. $(2014)$ \\
$\mathrm{InHg}$ & $10^{-5} \mathrm{M}$ & OC & nibbler fish & Yachiguchi et al. $(2014)$ \\
$\mathrm{InHg}$ & $10^{-5} \mathrm{M}$ & OB & nibbler fish & Yachiguchi et al. $(2014)$ \\
$\mathrm{TBT}$ & $10^{-10} \mathrm{M}$ & OB & goldfish & Suzuki et al. $(2006)$ \\
$\mathrm{TBT}$ & $10^{-8} \mathrm{M}$ & OB & nibbler fish & Suzuki et al. (2006) \\
$\mathrm{TBT}$ & $10^{-8} \mathrm{M}$ & OB & wasse & Suzuki et al. (2006)
\end{tabular}

Cd: cadmium; MeHg: methylmercury; InHg: inorganic mercury; TBT: tributyltin.

of tributyltin (TBT), an aquatic environmental pollutant used as a biocide in anti-fouling paint (Suzuki et al., 2006). Osteoblastic activity significantly decreased from the control values as a result of TBT treatment $\left(10^{-10}\right.$ to $10^{-5} \mathrm{M}$ ) at 6 hours of incubation (Suzuki et al., 2006). Judging from the comparison of heavy metals ( $\mathrm{Cd}$ and $\mathrm{MeHg}$ ) and TBT, the inhibitory effect on osteoblasts at 6 hours of incubation appears to have lower expression of MT mRNA in TBT-treated scales than in Cd- or MeHgtreated scales (Suzuki et al., 2006). Therefore, the toxicity of Gd to osteoblasts may be related to MT expression, as with TBT.

In Table 1, we summarized the comparison of toxicity to osteoclasts and osteoblasts of fish scales. Toxicity of $\mathrm{Gd}$ to osteoclasts was higher than those of $\mathrm{MeHg}$ and $\mathrm{InHg}$. The toxicity of $\mathrm{Gd}$ was comparable to that of $\mathrm{Cd}$. $\mathrm{Gd}$ inhibited osteoblastic activity and was toxic to osteoblasts. This inhibition of Gd was almost equal to that of TBT. In addition, the sensitivity of $\mathrm{Gd}$ in osteoclasts and osteoblasts was varied among respective goldfish. In the present study, female goldfish were used because sensitivity of osteoblastic and osteoclastic activities of scales to calcemic hormones was higher in mature females than in mature males (Suzuki et al., 2000; Yoshikubo et al., 2005). It seems that the sensitivity of $\mathrm{Gd}$ is related to the maturation of female goldfish. In the future, we are planning to study the correlation between sex hormones and Gd toxicity.

Gd was present in recycled water in which the stable organic Gd complexes pass through several sewage treatment plants without being significantly decomposed (Knappe et al., 2005). Therefore, anomalously high concentrations of $\mathrm{Gd}$ in surface waters are of anthropogenic origin (Rogowska et al., 2018). Specifically, anthropogenic Gd was detected in surface waters (up to $1,100 \mathrm{ng} / \mathrm{L}$ ) and sediments (up to $90.5 \mu \mathrm{g} / \mathrm{g}$ ) (Rogowska et al., 2018). Therefore, aquatic plants, fungi, small planktonic crustaceans, and freshwater fish (Cyprinus carpio) were able to take up anthropogenic $\mathrm{Gd}$ from the polluted water and it accumulated in their bodies (Rogowska et al., 2018). Furthermore, in the present study, we demonstrated that a level of $\mathrm{Gd}$ that is quite low $\left(10^{-13}\right.$ to $\left.10^{-11} \mathrm{M}\right)$ has toxicity for bone metabolism in goldfish. Thus, we strongly believe that anthropogenic Gd has toxicity for aquatic animals and we must consider a $\mathrm{Gd}$ risk assessment to protect the polluted aquatic environment.

\section{Conclusion}

We are the first to indicate the toxicity of Gd on fish bone metabolism using TRAP and ALP enzyme activities. We found that the detection limit of $\mathrm{Gd}$ in our bioassay was $10^{-13} \mathrm{M}$. Therefore, our assay system is quite effective as a biosensor of $\mathrm{Gd}$. In addition, $\mathrm{Gd}$ toxicity to osteoblasts appears to be higher than that to $\mathrm{Cd}, \mathrm{MeHg}$ and $\mathrm{InHg}$ and equal to TBT. The toxicity of $\mathrm{Gd}$ in osteoclasts was comparable to that of $\mathrm{Cd}$. Gd used for clinical diagnoses in MRI is released into environmental waters without a specific recycling process. Gd was found in tap water samples in the area of Berlin, Germany. Up to $18 \mathrm{ng} / \mathrm{L}$ of anthropogenic $\mathrm{Gd}$ on top of the geogenic background of $0.54 \mathrm{ng} / \mathrm{L}$ could be determined (Kulaksiz and Bau, 2011). Recently, the need for $\mathrm{Gd}$ risk assessments for human health has been emphasized (Fraum et al., 2017; Gwenzi et al., 2018). In addition to the risk to human, we should also emphasize Gd risk assessment to protect the ecosystem in the aquatic environment.

\section{Funding Information}

This study was supported partly by grants to N.S. (Kanazawa University CHOZEN Project) and to A.K.S. (the Cooperative Research Program of the Institute of Nature and Environmental Technology, Kanazawa University, No. 18035). 


\section{Authors' Contributions}

N. Suzuki and A. Hattori: Designed this study.

N. Suzuki, K. Watanabe, A. Sekimoto, M.I. Zanaty, T. Sekiguchi, Y. Kitani, H. Matsubara and M. Urata: Analyzed the data and wrote the paper including graph and table preparation.

A. Hattori and A.K. Srivastav: Supervised this work.

\section{Conflict of Interest}

The authors have no conflicts of interest to declare.

\section{Ethics}

All experimental procedures were conducted in accordance with the Guide for the Care and Use of Laboratory Animals of Kanazawa University.

\section{References}

Bereiter-Hahn, J. and L. Zylberberg, 1993. Regeneration of teleost fish scale. Comp. Biochem. Physiol. Part A, 105: 625-641.

DOI: 10.1016/0300-9629(93)90262-3

Birka, M., C.A. Wehe, O. Hachmöller, M. Sperling and U. Karst, 2016. Tracing gadolinium-based contrast agents from surface water to drinking water by means of speciation analysis. J. Chromatogr. A, 1440: 105-111. DOI: 10.1016/j.chroma.2016.02.050

Braun, M., G. Zavanyi, A. Laczovics, E. Berényi and S. Szabó, 2018. Can aquatic macrophytes be biofilters for gadolinium based contrasting agents? Water Res., 135: 104-111.

DOI: 10.1016/j.watres.2017.12.074

Dimai, H.P., T.A. Linkhart, S.G. Linkhart, L.R. Donahue and W.G. Beamer et al., 1998. Alkaline phosphatase levels and osteoprogenitor cell numbers suggest bone formation may contribute to peak bone density differences between two inbred strains of mice. Bone, 22: 211-216.

DOI: $10.1016 / \mathrm{S} 8756-3282(97) 00268-8$

Fraum, T.J., D.R. Ludwig, M.R. Bashir and K.J. Fowler, 2017. Gadolinium-based contrast agents: A comprehensive risk assessment. J. Magn. Reson. Imaging, 46: 338-353. DOI: 10.1002/jmri.25625

Gwenzi, W., L. Mangori, C. Danha, N. Chaukura and N. Dunjana et al., 2018. Sources, behaviour, and environmental and human health risks of hightechnology rare earth elements as emerging contaminants. Sci. Total Environ., 636: 299-313. DOI: $10.1016 /$ j.scitotenv.2018.04.235

Knappe, A., P. Möller, P. Dulski and A. Pekdeger, 2005. Positive gadolinium anomaly in surface water and ground water of the urban area Berlin, Germany. Chemie der Erde - Geochemistry, 65: 167-189. DOI: 10.1016/j.chemer.2004.08.004
Kulaksız, S. and M. Bau, 2011. Anthropogenic gadolinium as a microcontaminant in tap water used as drinking water in urban areas and megacities. Appl. Geochem., 26: 1877-1885.

DOI: 10.1016/j.apgeochem.2011.06.011

Lehane, D.B., N. Mckie, R.G.G. Russell and I.W. Henderson, 1999. Cloning of a fragment of the osteonectin gene from goldfish, Carassius auratus: Its expression and potential regulation by estrogen. Gen. Comp. Endocrinol., 114: 80-87.

DOI: $10.1006 /$ gcen.1998.7237

Martino, C., C. Costa, M.C. Roccheri, D. Koop and R. Scudiero et al., 2018. Gadolinium perturbs expression of skeletogenic genes, calcium uptake and larval development in phylogenetically distant sea urchin species. Aquat. Toxicol., 194: 57-66. DOI: $10.1016 /$ j.aquatox.2017.11.004

Martino, C., R. Chiarelli, L. Bosco and M.C. Roccheri, 2017. Induction of skeletal abnormalities and autophagy in Paracentrotus lividus sea urchin embryos exposed to gadolinium. Mar. Environ. Res., 130: 12-20.

DOI: 10.1016/j.marenvres.2017.07.007

Möller, P., T. Paces, P. Dulski and G. Morteani, 2002. Anthropogenic Gd in surface water, drainage system and the water supply of the city of Prague, Czech Republic. Environ. Sci. Technol., 36: 2387-2394. DOI: $10.1021 / \mathrm{es} 010235 \mathrm{q}$

Mugiya, Y. and N. Watabe, 1977. Studies on fish scale formation and resorption II: Effect of estradiol on calcium homeostasis and skeletal tissue resorption in the goldfish, Carassius auratus and the killifish, Fundulus heteroclitus. Comp. Biochem. Physiol. Part A, 57: 197-202.

DOI: 10.1016/0300-9629(77)90455-8

Nishimoto, S.K., N. Araki, F.D. Robinson and J.H. Waite, 1992. Discovery of bone $\gamma$-carboxyglutamic acid protein in mineralized scales. J. Biol. Chem., 267: 11600-11605.

DOI: 10.1111/j.2040-1124.2010.00046.x

Rogowska, J., E. Olkowska, W. Ratajczyk and L. Wolska, 2018. Gadolinium as a new emerging contaminant of aquatic environments. Environ. Toxicol. Chem., 37: 1523-1534.

DOI: $10.1002 /$ etc.4116

Saitoh, M., R. Kuroda, Y. Muranaka, N. Uto and J. Murai et al., 2010. Asymmetric inhibition of spicule formation in sea urchin embryos with low concentrations of gadolinium ion. Develop. Growth Differ., 52: 735-746. DOI: $10.1111 /$ j.1440-169X.2010.01210.x 
Sherry, A.D., P. Caravan and R.E. Lenkinski, 2009. Primer on gadolinium chemistry. J. Mag. Reson. Imaging 30: 1240-1248. DOI: 10.1002/jmri.21966

Suda. T., N. Takahashi, N. Udagawa, E. Jimi and M.T. Gillespie et al., 1999. Modulation of osteoclast differentiation and function by the new members of the tumor necrosis factor receptor and ligand families. Endocrine Rev., 20: 345-357.

DOI: $10.1210 /$ edrv.20.3.0367

Suzuki, N. and A. Hattori, 2002. Melatonin suppresses osteoclastic and osteoblastic activities in the scales of goldfish. J. Pineal Res., 33: 253-258.

DOI: 10.1034/j.1600-079X.2002.02953.x

Suzuki, N. and A. Hattori, 2003. Bisphenol A suppresses osteoclastic and osteoblastic activities in the cultured scales of goldfish. Life Sci., 73: 2237-2247. DOI: 10.1016/S0024-3205(03)00603-9

Suzuki, N., K. Yachiguchi, K. Hayakawa, K. Omori and K. Takada et al., 2011. Effects of inorganic mercury on osteoclasts and osteoblasts of the goldfish scales in vitro. J. Fac. Agr. Kyushu Univ., 56: 47-51.

Suzuki, N., M. Yamamoto, K. Watanabe, A. Kambegawa and A. Hattori, 2004. Both mercury and cadmium directly influence calcium homeostasis resulting from the suppression of scale bone cells: The scale is a good model for the evaluation of heavy metals on bone metabolism. J. Bone Miner. Metab., 22: 439-446. DOI: $10.1007 / \mathrm{s} 00774-004-0505-3$

Suzuki, N., T. Suzuki and T. Kurokawa, 2000. Suppression of osteoclastic activities by calcitonin in the scales of goldfish (freshwater teleost) and nibbler fish (seawater teleost). Peptides, 21: 115124. DOI: 10.1016/S0196-9781(99)00181-3
Suzuki, N., T.M. Tabata, M.A. Kambegawa, A.K. Srivastav and A. Shimada et al., 2006. Tributyltin inhibits osteoblastic activity and disrupts calcium metabolism through an increase in plasma calcium and calcitonin levels in teleosts. Life Sci., 78: 2533-2541. DOI: $10.1016 /$ j.lfs.2005.10.004

Telgmann, L., M. Sperling and U. Karst, 2013. Determination of gadolinium-based MRI contrast agents in biological and environmental samples: A review. Anal. Chim. Acta, 764: 1-16. DOI: 10.1016/j.aca.2012.12.007

Thamamongood, T.A., R. Furuya, S. Fukuba, M. Nakamura and N. Suzuki et al., 2012. Expression of osteoblastic and osteoclastic genes during spontaneous regeneration and autotransplantation of goldfish scale: A new tool to study intramembranous bone regeneration. Bone, 50: 1240-1249. DOI: 10.1016/j.bone.2012.03.021

Vaes, G., 1988. Cellular biology and biochemical mechanism of bone resorption: A review of recent developments on the formation, activation and mode of action of osteoclasts. Clin. Orthop. Relat. Res., 231: 239-271.

Yachiguchi, K., T. Sekiguchi, M. Nakano, A. Hattori and M. Yamamoto et al., 2014. Effects of Inorganic mercury and methylmercury on osteoclasts and osteoblasts in the scales of the marine teleost as a model system of bone. Zool. Sci., 31: 330-337. DOI: $10.2108 / \mathrm{zs} 130265$

Yoshikubo, H., N. Suzuki, K. Takemura, M. Hoso and S. Yashima et al., 2005. Osteoblastic activity and estrogenic response in the regenerating scale of goldfish, a good model of osteogenesis. Life Sci., 76: 2699-2709. DOI: 10.1016/j.lfs.2004.10.063 